\title{
AUTOLOGOUS BLOOD INJECTION INTRACORONARY ARTERY FOR TREATING SLOW-FLOW AND NO-REFLOW IN ACUTE CORONARY SYNDROME RELATED TO PRIMARY PCI
}

Lam Truong Hoai ${ }^{1}$, Hung Nguyen Duc ${ }^{1}$, Duy Nguyen Xuan ${ }^{1}$, and Long Nguyen Tuan ${ }^{1}$

${ }^{1}$ Tam Anh Hospital

July 9, 2021

\begin{abstract}
Slow flow and no-reflow phenomenon are taken to sudden loss of coronary artery flow, typically after stenting or angioplasty in primary PCI. Otherwise conventional therapy, we report a technique, which autologous blood into intracoronary to supply oxygen and break process thrombosis results in successfully management no-reflow in primary PCI
\end{abstract}

TITLE:

AUTOLOGOUS BLOOD INJECTION INTRACORONARY ARTERY FOR TREATING SLOW-FLOW AND NO-REFLOW IN ACUTE CORONARY SYNDROME RELATED TO PRIMARY PCI

Short title: Autologous blood injection intracoronary artery

The author's name :

MD. Lam Truong Hoai

Affiliation: Tam Anh Hospital.

Mail: truonglamcs@gmail.com

Address: Hanoi Capital, Vietnam

MD. Duy Nguyen Xuan

Affiliation: Tam Anh Hospital

Mail: nguyenduyk41qy@gmail.com

Address: Hanoi Capital, Vietnam

MD. Hung Nguyen Duc

Affiliation: Tam Anh Hospital

Mail: hungcathlab@gmail.com

Address: Hanoi Capital, Vietnam

MD. Long Nguyen Tuan 
Affiliation: Tam Anh Hospital

Mail: tuanlong1706@gmail.com

Address: Hanoi Capital, Vietnam

\title{
Corresponding author's Full name: Lam Truong Hoai
}

Affiliation: Tam Anh Hospital

Address: Hanoi Capital, Vietnam

Email: truonglamcs@gmail.com

Tel : +84036875579

Keywords: Slow flow \& no-Reflow, acute coronary syndrome, acute crisis thrombosis, balloon angioplasty, autologous blood injection

Abbreviation: percutaneous coronary intervention (PCI), Acute coronary syndrome (ACS), Intact related artery(IRA), Right coronary artery (RCA), Posterior left ventricular (PLV), Ventricular tachycardia (Vtach), Blood pressure (BP), ST-elevation myocardial infarction (STEMI), Clinical key message:

Management of the slow-flow and no-reflow when the conventional therapy isn't already.

That may be a new technique that considers when hemodynamic unstable and isn't suitable with the medication especially without IIb/IIIa inhibitor

\begin{abstract}
Slow flow and no-reflow phenomenon are taken to sudden loss of coronary artery flow, typically after stenting or angioplasty in primary PCI. Otherwise conventional therapy, we report a technique, which autologous blood into intracoronary to supply oxygen and break process thrombosis results in successfully management no-reflow in primary PCI in ACS
\end{abstract}

\section{Introduction}

Early revascularization of an intact related artery ( IRA) plays an important role in ACS to prevent myocardium injury. With all the recent advances in equipment and techniques, revascularization has become a faster and good result. However, the no-reflow phenomenon occurs in a considerable number of patients undergoing primary PCI ranging between $12 \%$ and $32.8 \%(1)$. This phenomenon is associated with arrhythmias, poor in-hospital survival, and poor one-year survival. Intracoronary vasodilators such as verapamil, nitroprusside, or adenosine are being administered for the treatment of no-reflow via the microcatheter. But sometimes distal flow restoration is not satisfactory especially in patients with TIMI 0 flow because of hemodynamic unstable and heart shock result in less effective medication lead to the circuit pathology is that no flow, thrombosis, heart shock, arrhythmias with consequence death. The use of blood with high O2 saturation pump into the intracoronary to supply oxygen for the myocardium while the no-reflow phenomenon occurs, and this helps to maintain flow in the vessel to prevent thrombosis. We report a clinical case using the self blood pump into the coronary artery to treat no flow intracoronary in ACS patients with primary PCI successfully.

\section{Presentation}

A 61-year-old man with a history of hypertension was admitted to the hospital because of severe chest pain. In the emergency department, the patient suddenly cardiac arrest, and chest compressions immediately with Adrenaline $1 \mathrm{mg} / 1 \mathrm{ml} \times 5$ ampoules, after that monitoring showed Vtach and defibrillation with 200J. ECG showed BAV III, ST elevated in DII, DIII, aVF, and heart rate was $40 \mathrm{bpm}$ (figure 1). Blood pressure was $90 / 60 \mathrm{mmHg}$ with Noreadrenalin $0.1 \mathrm{mcg} / \mathrm{kg} / \mathrm{h}$, Dobutamine $10 \mathrm{mcg} / \mathrm{kg} / \mathrm{h}$ and mechanical ventilation. Patient transferred to cath lab immediately with totally medication which was, Lidocaine $40 \mathrm{mg} / 2 \mathrm{ml} \times 2$ ampoules, Magnesisulfat 15\%x10ml x 2 ampoules, Lovenox 0,5mg/kg, Aspirin x 300mg, Ticagrelor 180mg, 
Rosuvastatin 40mg. A temporary pacemaker was implanted on the right femoral vein with HR 80 bpm, output $3 \mathrm{mv}$, sensing $3 \mathrm{mv}$. The angiography on the right femoral artery showed proximal RCA occlusion (figure 2A). The thrombus aspiration device had been used, which wasn't effective. Balloon angioplasty which complaint ballon $2.5 \times 20 \mathrm{~mm}$ was used, angiogram showed RCA TIMI III flow and lesion were RCA I-II and RCA III with $95 \%$ and $90 \%$ irrespectively. Stents were implanted with DES 3.5x40mm (20atm) for RCA I-II, and DES $2.75 \times 40 \mathrm{~mm}$ (12atm) for RCA III-PLV. The hemodynamic was stable BP 100/60mmHg (with noradrenaline and dobutamine) and the TIMI III flow was showed on the angiogram. After 5 minutes the angiogram showed thrombus in the proximal RCA (Figure 2B) (ACT was 230sec). With the mud thrombosis which the thrombus aspiration device wasn't effective, we decided active balloon angioplasty with a compliant balloon $(3.5 \times 20 \mathrm{~mm})$ to maintain the flow but as a result slow-flow on the angiogram (TIMI I), which may be microvascular constriction result in hemodynamic unstable and arrhythmia (Vtach or Vfib). The medication was indicated which wasn't suitable because of low BP and arrhythmia, include Adenosine, Nitroglycerin. To maintain flow in RCA we decided active angioplasty balloon and the use of blood with high $\mathrm{O} 2$ saturation pump into the intracoronary to supply oxygen for the myocardium while the no-reflow phenomenon occurs through a contralateral femoral artery. Using syringe $10 \mathrm{ml}$ pumped through thrombus aspiration device, hand force just enough to reduce erythrocyte rupture, with a frequency of about 5 pumps/min, active angioplasty balloon every 5 minutes to maintain open vessel lumen and maintain flow. Thrombus aspiration still measures coronary perfusion pressure which demonstrates perfusion in the coronary artery while couldn't measure pressure through guiding catheter. The patient became more stable, which arrhythmia and pressure drop weren't apparent while we did the procedure. After 1 hour of active balloon angioplasty and pump blood with high O2 saturation, the angiogram showed TIMI III flow and thrombus apparent, hemodynamically stable, the patient was transferred to CCU for monitoring and treatment. After 3 days of wearing off ventilators and vasoconstriction. After a total of 10 days, the patient was discharged with a stable condition.

\section{Discussion}

Originally, it was thought prolonged ischemia and extensive myocardial damage led to microvascular (capillary bed) damage, or microvascular vasoconstriction resulting in incomplete reperfusion, other factors have been thought to play an important role in the development of no-reflow, Platelets may be implicated in slow flow and no-reflow through several mechanisms, including micro-vascular vasoconstriction or obstruction by platelet aggregates. Although the exact mechanism of no-reflow remains unknown, it is most likely complex and multi-factorial. The selective use of Glycoprotein IIb/IIIa inhibitors and thrombectomy devices during the intervention may be also appropriate in the selected case, but the mud thrombus almost failed with the thrombectomy device, and Glycoprotein IIb/IIIa lack of randomized controlled clinical trials remains a limitation. Unfortunately, In Vietnam, the Glycoprotein IIb/IIIa hasn't already. However, no-reflow can still occur even under the best-provided care, which emphasizes the need for more specific mechanical and/or medical treatments. This is particularly true in the setting of an acute STEMI. Various vasodilators have been shown to affect some cases of success including nicardipine, nitroprusside, and verapamil, but the longeracting drugs are somewhat limited by significant hemodynamic unstable and arrhythmia, negative inotropy, which are of particular importance in the acutely ischemic heart. Two drugs in particular (nitroprusside and adenosine) have been studied as possible adjuncts to reperfusion therapy in some trials $(2,3)$ but the effect isn't strong enough to succeed in all cases especially in an unstable hemodynamic and significantly arrhythmias situation while continuously autologous blood injection into the intracoronary artery through aspiration thrombus device is an important role to good choice to keep flowing compensate flow disturbance. Autologous blood injection which is high $\mathrm{O} 2$ saturation into the coronary artery with the pressure and velocity stable to demand myocardium to reduce the myocardium injury when slow flow and no-reflow occurred, which is promised technique. In addition, active balloon angioplasty to break thrombus formation and fibrin results in decrease crisis thrombosis and maintain flow. As a result, the coronary flow is preserved. Keep do it until the crisis thrombosis pass away and the flow is improved. The time to pass to crisis thrombosis is important due to such thrombin activity and fibrin polymerization fronts typically travel slowly at a rate of $3 \mathrm{~mm}$ in $60 \mathrm{~min}$, consistent with simulation(4). Slow traveling wave propagation to the physics in stagnant 
blood clotting, while blood injection can warrant the pressure and velocity of flow with high oxygen while the patient suffers from cardiac shock, is that the medication show less effect. Moreover, in a critical setting, continuous autologous blood injection intracoronary artery can play an important role until all disturbance disappeared. Similar to ECMO and IABP are ensure hemodynamic and oxygen for myocardium by systemic support, and autologous blood injection to supply for local ischemic myocardium. Virchow's triad shows the 3rd factor is that vessel injury, stasis of blood, and hypercoagulability of blood, which is correlative with the mechanism of crisis thrombosis after stenting due to STEMI, while blood artery can cover include stasis of blood by injecting blood into intracoronary with stable pressure and active balloon angioplasty to maintain flow, to supply oxygen for myocardium to reduce endothelium damages, necrosis, supplying more fibrinogen form another site where the crisis thrombosis doesn't occur. Moreover slow flow and no re-flow could preserve. This is the reason why we should keep do procedures while waiting for the flow preserve to become normal.

\section{Conclusion}

Autologous blood which high oxygen inject to the intracoronary artery and active balloon angioplasty is a role of treating slow flow \& no re-flow when the other conventional therapy isn't suitable especially in hemodynamic unstable and arrhythmias situation. Keep flowing and demand high blood oxygen to reduce endothelial damage and myocardium necrosis result in increase survival when crisis thrombosis and disturbance flow appearance.Funding: NoneDisclosures: None

\section{References}

1. Alidoosti M, Lotfi R, Lotfi-Tokaldany M, Nematipour E, Salarifar M, Poorhosseini H, et al. Correlates of the "No-Reflow" or "Slow-Flow" Phenomenon in Patients Undergoing Primary Percutaneous Coronary Intervention. J Tehran Heart Cent. 2018;13(3):108-14.

2. Ross AM, Gibbons RJ, Stone GW, Kloner RA, Alexander RW. A randomized, double-blinded, placebocontrolled multicenter trial of adenosine as an adjunct to reperfusion in the treatment of acute myocardial infarction (AMISTAD-II). J Am Coll Cardiol. 2005;45(11):1775-80.

3. Parikh KH, Chag MC, Shah KJ, Shah UG, Baxi HA, Chandarana AH, et al. Intracoronary boluses of adenosine and sodium nitroprusside in combination reverses slow/no-reflow during angioplasty: a clinical scenario of ischemic preconditioning. Can J Physiol Pharmacol. 2007;85(3-4):476-82.

4. Diamond SL. Systems Analysis of Thrombus Formation. Circ Res. 2016;118(9):1348-62.

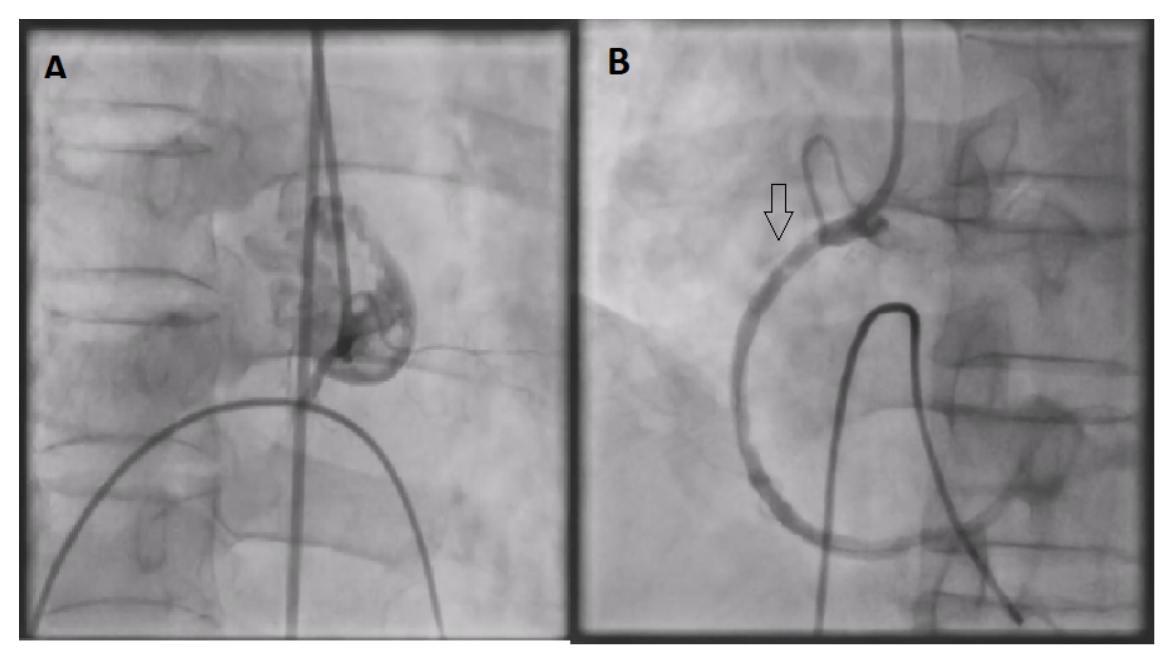




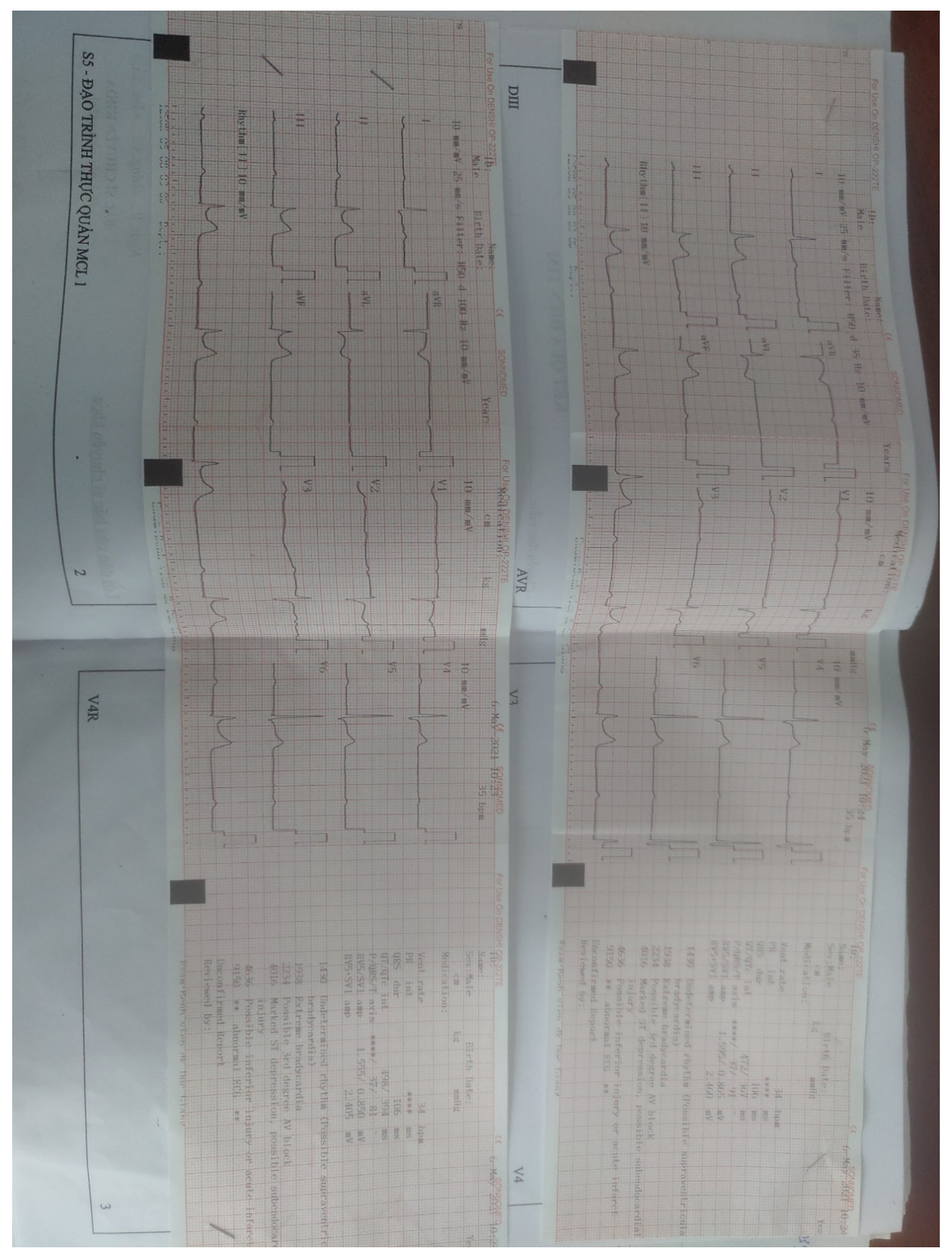

\section{Hosted file}

Figure 1,2.docx available at https://authorea.com/users/424725/articles/529754-autologousblood-injection-intracoronary-artery-for-treating-slow-flow-and-no-reflow-in-acutecoronary-syndrome-related-to-primary-pci 